\title{
About the Origins of the General Theory of Relativity: Einstein's Search for the Truth.
}

\author{
Matthew Trainer \\ Kelvin Building, \\ Department of Physics and Astronomy, \\ University of Glasgow, \\ Glasgow G12 8QQ, UK
}

email: m.trainer@physics.gla.ac.uk

\begin{abstract}
On the 20th June 1933 Professor Einstein addressed a large and enthusiastic audience in the Victorian Gothic Bute Hall of the University of Glasgow. Einstein spoke 'About the Origins of the General Theory of Relativity'. In 1905 Einstein had changed the face of physics forever with the publication of his radical new ideas on special relativity. His general theory of relativity was introduced to the world in 1915. However in 1933, Einstein faced another challenge - survival in a world of change. This paper explores Einstein's fascinating address to the Glasgow audience in that year.
\end{abstract}

\section{Introduction}


During 1933 Einstein visited the United Kingdom, lecturing in London, Oxford and Glasgow. Einstein's address at Glasgow University took place in the evening of the 20th June 1933 in the grand Bute Hall [1]. Figure 1 shows an exterior view of this Victorian Gothic hall. The address was 'About the Origins of the General Theory of Relativity' and was funded by the G. A. Gibson Foundation [2]. General relativity was Einstein's enigmatic theory of gravitation and his oration received a resounding applause. On the following day, the Principal, Sir Robert Rait honoured Einstein with a Doctor of Laws (LL.D.) degree [3].

This paper aims to examine the origins of the general theory of relativity as related by Professor Einstein in his address to Glasgow University [4]. The author has expanded on the various themes on the history and theory to give the reader a fuller understanding of Einstein's deduction of his definitive theory.

\section{Einstein's Address - Searching for the Truth}

\subsection{Fascinating Idea}

After Einstein had formulated the special theory of relativity for inertial systems in 1905 he wondered whether a more general theory existed that would include accelerated systems and Newton's law of gravity. Einstein mentioned that he was familiar with the work of Ernst Mach. Mach (1838 - 1916), Professor of Experimental Physics at the Charles University in Prague, had criticized Newton's concept of motion of a body with respect to the mathematical abstraction of absolute space. He conceived the hypothesis of motion relative to a more material system such as distant stars. Mach had the intriguing idea that the inertia of any body is the result of the interaction of that body with the rest of the 
bodies in the universe [5]. Einstein was fascinated by Mach's idea on inertia and stated in the address 'This idea fascinated me; but it did not provide a basis for a new theory' [4].

Einstein endeavoured to find a gravitational 'field law'. For he had established in his special theory of relativity that Newton's law of instantaneous action at a distance was no longer valid. His theory had abolished the idea of simultaneity. For now it was clear to Einstein that events that were simultaneous in one frame of reference were not necessarily simultaneous in another frame.

Einstein decided to start from the Laplace scalar potential of gravity. The French mathematician Pierre-Simon Laplace $(1749$ - 1827) conceived his equation more than a century earlier. The scalar form of Laplace's equation is the partial differential equation

$$
\nabla^{2} \psi=0
$$

where $\nabla^{2}$ is the Laplacian (or partial differential operator) for a scalar function $\psi$. Laplace's equation applies to regions of space outside matter [6]. Einstein's next step was to examine the mathematics of Siméon Denis Poisson $(1781-1840)$. Poisson used the gravitational potential approach to give an equation that could be solved under rather general conditions. Poisson's equation took the form of

$$
\nabla^{2} \psi=-4 \pi G \rho
$$

This equation applies for regions of space in which $\rho$ is the material density and $G$ is the Newtonian constant of gravitation [6].

Einstein went on to say that the law of motion of a particle in a gravitational field had to conform to the special theory of relativity but how to do this was not clear to him. 


\subsection{Grave Misgivings}

Einstein's investigations made him uneasy for 'According to the classical mechanics, the vertical acceleration of a body in a vertical field of gravitation was independent of the horizontal component of the velocity' [4]. However, in Einstein's special of relativity theory the vertical acceleration was not independent of the horizontal velocity. Indeed, Einstein's theory was not in agreement with the well-established empirical formula of the law of the equivalence of inertial and gravitational mass. This law, familiar to Galileo and Newton, stated that all bodies in a gravitational field were subject to the same acceleration. Einstein went on to say 'I wondered how this law could exist and believed that it held the key of the understanding of inertia and gravitation' [4].

Einstein mentioned that at that time he was not familiar with the 'beautiful experiments of Eötvös' [4]. These showed the equivalence of gravitational mass and inertial mass to a high degree of accuracy [7]. Lóránd Eötvös (1848 -1919), Professor of Experimental Physics at the University of Budapest, had studied the experiment of Armand Fizeau on the relative motion of a light source, an experiment that Einstein later discussed in his book on relativity [8]. It is believed that this experiment had been one of the first steps towards relativity theory.

\subsection{Extension of Principle}

It was now clear to Einstein that the special theory of relativity was inadequate to take account of the most fundamental property of gravitation - the principle of equivalence. In his address he stated 'I gave up, therefore, the attempt, which I have sketched above, to treat the problem of gravitation within the frame-work of 
the special theory of relativity' [4]. He believed that for a theory to be successful then the principle of equivalence should fit into it in a very simple and clear manner. So from 1908 to 1911 Einstein concentrated on obtaining a theory of gravitation by an extension of relativity theory to include non-uniform motions of the co-ordinate systems $[9,10]$.

It was now very clear to Einstein of the direction of his work as he stated 'The problem therefore was to find and to elaborate a theory expressed in equations which did not change their form for non-linear transformations of the coordinates' [4].

\subsection{A New Formulation}

In 1912 Einstein looked for a new formulation of the inertial principle that would become identical to Galileo's formulation in the absence of a gravitational field. Galileo formulated the concept of inertia: an object in a state of motion possesses an inertia that causes it to remain in that state of motion unless an external force acts on it. Einstein at this point modified Galileo's formulation and expressed it thus: 'a material particle on which no forces are acting, is represented in fourdimensional space by a straight line' [4].

Einstein mentioned the work of the mathematician Hermann Minkowski (1864 -1909) who recognized that the correct way to view special relativity was in terms of a four-dimensional space-time. This was a combination of the dimension of time with the three dimensions of space. In the special theory of relativity Minkowski had considered the space-time continuum as quasi-Euclidian. According to Minkowski, the square of the small length $d s$ of a line was a quadratic function of the differential coefficients of the coordinates $\left(x_{1}, x_{2}, x_{3}, x_{4}\right)$ 


$$
d s^{2}=d x_{1}^{2}+d x_{2}^{2}+d x_{3}^{2}+d x_{4}^{2}
$$

$x_{4}$ is an imaginary time variable $\sqrt{-1} c t$ which replaces the real time $t$ [8].

Now Euclidean geometry was based on flat surfaces but Einstein realized that space could be curved and so for a general theory of relativity the space-time continuum was non-Euclidean [8]. In fact Einstein's conclusion was that the bending of space and time by bodies caused gravity. Einstein soon found the mathematics to describe the bending of space-time 'Mathematically, this means that the physical four-dimensional continuum had a Riemann metric' [4]. In 1854 Bernhard Riemann (1826 -1866) introduced the field of Riemannian geometry into his lectures at the University of Göttingen [11]. With these mathematical methods he was able to describe the geometry of curved surfaces in any dimension. Now if the square of the small length $d s$ is given by a quadratic differential equation

$$
d s^{2}=A d x^{2}+2 B d x d y+C d y^{2}
$$

in the surface coordinates where $A, B$ and $C$ are certain functions of the coordinates then $d s^{2}$ is described as a Riemann metric and the corresponding surface is Riemannian. An interesting point is that the Reimannian metric is locally Euclidean [12].

\subsection{The Solution}

Einstein found a workable basis for the general theory of relativity in Riemannian geometry. However he stated that two problems remained to be solved:

(1) 'How can we translate a field law given us in the terminology of the special theory of relativity into a Riemann metric?' 
(2) 'What are the differential expressions which enter into the law holding for the Riemann metric?' [4]

Einstein mentioned that from 1912 to 1914 he worked with his former classmate Marcel Grossmann. Professor Grossmann (1878 - 1936) pointed out that the mathematical methods for solving the first problem lay in the absolute differential calculus of Ricci-Curbastro and Levi-Civita [13]. The Italian mathematicians Gregorio Ricci-Curbastro (1853 - 1925) and Tullio Levi-Civita (1873 - 1941) introduced their mathematical methods in the paper 'Methods of Absolute Differential Calculus and their Application' published in 1901 [14].

Grossmann explained to Einstein that Riemann had already worked out the mathematical methods required for the solution of the second problem. These took the form of Riemann curvature tensors [13]. The partnership of Einstein and Grossmann produced two papers, 'Outline of a Generalised Theory of Relativity and of a Theory of Gravitation' in 1913 [15] and 'Covariance Properties of the Field Equations of the Theory of Gravitation Based on the Generalised Theory of Relativity' in 1914 [16]. By 1913 they had laid out most of the elements of the general theory of relativity but had to settle for field equations of limited covariance [13]. However, to comply with the principle of general covariance the laws of physics should take the same form in all coordinate systems. It was important for Einstein to obtain complete covariance of the field equations in his general theory of relativity. He wanted equations that retained the same form regardless of the frame of reference being used, whether it was inertial or accelerating. At this stage Einstein put aside the Riemann curvature approach and his research went off in a different direction 'we failed to recognise that they were physically applicable' [4]. 


\subsection{Searching in the Dark}

Einstein paid dearly for his change of direction for in the address he conveyed to the audience 'These were errors in thinking, which caused me two years of hard work before at last in 1915 I recognised them as such and returned penitently to the Riemann curvature which enabled me to find the relation to the empirical facts of astronomy' [4]. After Einstein was in the correct mode of thought his boldest dreams came true. The final form of the field equations appeared in the 1915 paper 'The Field Equations of Gravitation' presented to the Prussian Academy of Sciences in Berlin [17]. As it is a tensor equation, the Einstein field equation is usually written out in terms of its components

$$
G_{\mu v}=\frac{8 \pi G}{c^{4}} T_{\mu v}
$$

where $G_{\mu v}$ are the components of the Einstein tensor, $G$ is the gravitational constant, $c$ is the speed of light and $T_{\mu \nu}$ are the components of the stress-energy tensor. Also

$$
G_{\mu v}=R_{\mu v}-\frac{1}{2} R g_{\mu v}
$$

where $G_{\mu \nu}$ are the components of the Einstein tensor, $R_{\mu \nu}$ are the components of the Ricci tensor, $R$ is the Ricci scalar and $g_{\mu \nu}$ are the components of the metric tensor [18].

Einstein's final and uplifting words in his address were 'But the years of searching in the dark for a truth that one feels but cannot express, the intense desire and the alternations of confidence and misgiving, until one breaks through to clarify and understanding, is only known to him, who has himself experienced $i t^{\prime}[4]$. 


\section{Conclusions}

Einstein's address was most certainly a memorable occasion for Glasgow University and especially for the department of Natural Philosophy (now Physics and Astronomy). No doubt, because of the time (20 minutes) allocated to him, Einstein emitted to mention many scientists and mathematicians who had played a role in the development of the theory of relativity. To name a few: David Hilbert (1862 - 1943) [19], Hendrik Lorentz (1853-1928), Jules Henri Poincaré (1854 1912) and Max Planck (1858-1947). However, the reality is that scientific endeavour is the sum of the work of many creative minds but it often takes one person to bring all the parts together to make the definitive theory. In Einstein's own words at the start of his Glasgow address: 'to write the history of the work of another requires an understanding of his mental processes which can be better achieved by professional historians '[4].

\section{Acknowledgement}

The author would like to express thanks to the University of Glasgow Library, Physics and Astronomy, the Hunterian Museum and the Mitchell Library for access to archives and documents. This paper was written as participation in the centenary of Einstein's miraculous year 1905 and World Year of Physics 2005.

\section{References}

[1] The Relativity Theory, Professor Einstein describes his Work 21st June 1933

(Mitchell Library Glasgow: Glasgow Herald newspaper page 11 column a) 
[2] George Alexander Gibson was Professor of Mathematics, University of Glasgow from 1909 to 1927 . In 1928 his friends raised a fund to endow a lecture on the history of mathematics to be delivered approximately every four years. [3] Honorary Degrees, Professor Einstein LL.D. 22nd June 1933 (Mitchell Library Glasgow: Glasgow Herald newspaper page 5 column a) [4] Einstein A 1933 About the Origins of the General Theory of Relativity (University of Glasgow: MS general 1314, a typescript amended in Einstein's handwriting)

[5] Goenner H 1970 Mach's Principle and Einstein's Theory of Gravitation Boston Studies in the Philosophy of Science VI 200-15 (Dordrecht: Reidel) [6] Chandrasekhar S 1979 Einstein and general relativity: Historical perspectives Am. J. Phys. 47 212-7

[7] Fischbach E and Aronson S H 1986 Reanalysis of the Eötvös Experiment Phys. Rev. Lett. 56 3-6

[8] Einstein A 1920 The Theory of Relativity (London: Meuthen \& Co. Ltd.) translator Robert W Lawson

[9] Einstein A 1989 The Swiss Years: Writings, 1909-1911 (The Collected Papers of A Einstein vol 3) ed J Stachel (Princeton NJ: Princeton University Press) [10] Einstein A 1989 The Swiss Years: Writings, 1900-1909 (The Collected Papers of A Einstein vol 2) ed J Stachel (Princeton NJ: Princeton University Press)

[11] Rindler W 1994 General relativity before special relativity: An unconventional overview of relativity theory Am. J. Phys. 62 887-93 [12] Rindler W 1977 Essential Relativity (New York: Springer-Verlag) 2nd edition pp110-4 
[13] Einstein A 1989 The Berlin Years: Writings, 1914-1917 (The Collected Papers of A Einstein vol 6) ed J Stachel (Princeton NJ: Princeton University Press)

[14] Ricci G and T Levi-Cività 1901 Méthodes de calcul différentiel absolu et leurs applications Mathematische Annalen 54 125-201.

[15] Einstein A and Grossmann M 1913 Entwurf einer verallgemeinerten Relativitätstheorie und einer Theorie der Gravitation (Leipzig: B G Teubner) [16] Einstein A and Grossmann M 1914 Kovarianzeigenschaften der Feldgleichungen der auf die verallgemeinerte Relativätstheorie gegründeten Gravitationstheorie Zeitschrift fur Mathematik und Physik 63 215-25 [17] Einstein A 1915 Die Feldgleichungen der Gravitation K. Preussische Akademie der Wissenschaften. Sitzungsberichte 844-47

[18] Price R H 1982 General relativity primer Am. J. Phys. 50 300-29

[19] Mehra J 1974 Einstein, Hilbert and The Theory of Gravitation (Boston: D. Reidel Publishing Company) 


\section{Captions}

Figure 1. Bute Hall where Einstein addressed a Glasgow audience in 1933. 


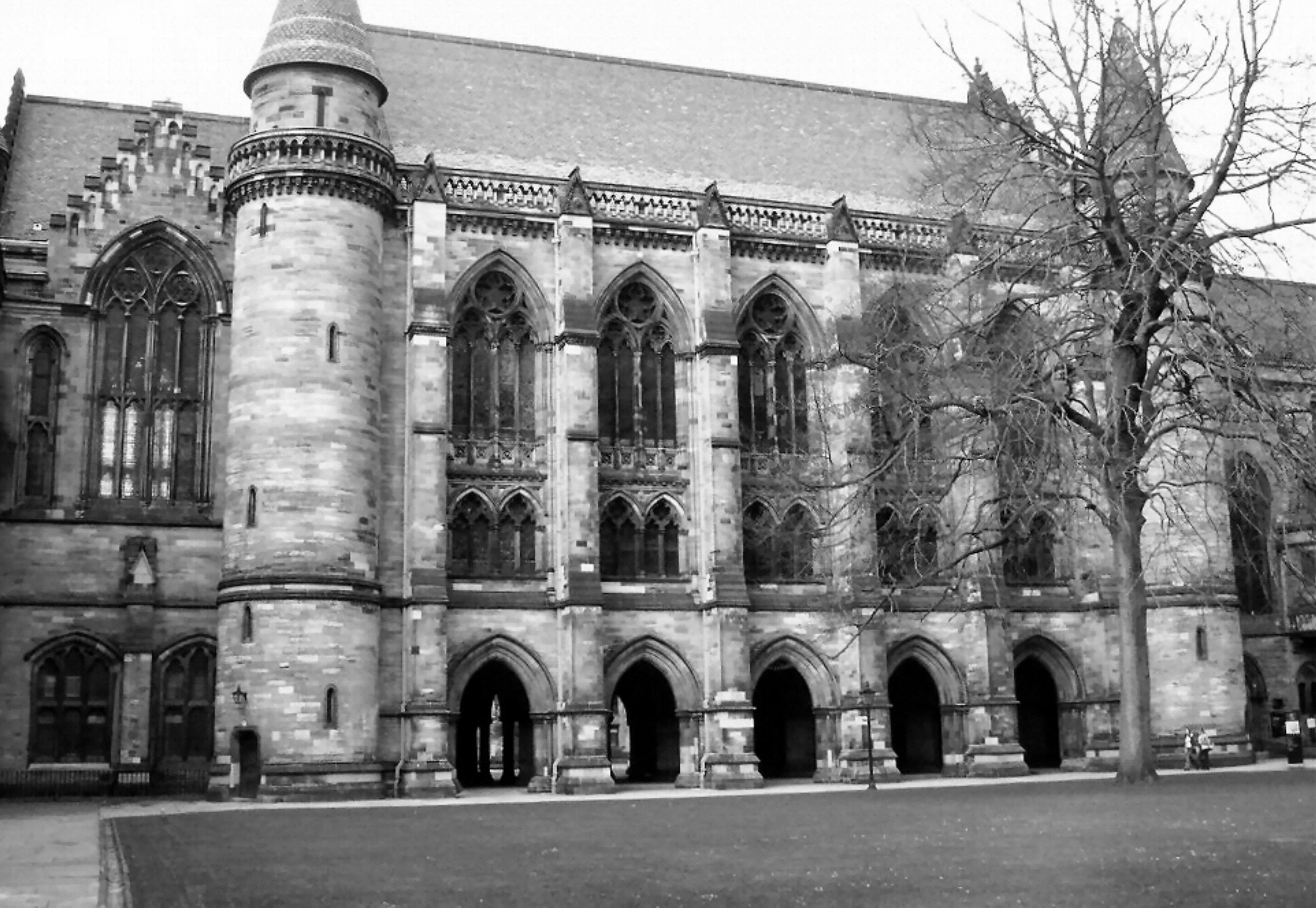

